\title{
MÉTODOS DE ESTIMAÇÃO DE COMPONENTES DE VARIÂNCIA EM MODELOS MISTOS DESBALANCEADOS'
}

\author{
Sandra Denisen do Rocio Marcelino ${ }^{2,5 *}$; Antonio Francisco lemma ${ }^{3,4}$ \\ ${ }^{2}$ Pós-Graduanda do Depto. de Ciências Exatas - USP/ESALQ. \\ ${ }^{3}$ Depto. de Ciências Exatas - USP/ESALQ, C.P. 9 - CEP: 13418-900 - Piracicaba, SP. \\ ${ }^{4}$ Centro de Ensino Superior de São Carlos, C.P. 307 - CEP: 13570-300 - São Carlos, SP. \\ ${ }^{5}$ Bolsista CNPq. \\ *Autor correspondente <sdrmarce@ig.com.br>
}

\begin{abstract}
RESUMO: A estimação dos componentes de variância em modelos mistos tem merecido, nas últimas décadas, atenção especial dos pesquisadores. Sendo assim, vários métodos de estimação têm sido propostos, e diante de tal disponibilidade, escolher um deles pode não ser uma questão trivial. Nesse contexto, buscando fornecer aos usuários dos métodos de estimação de componentes de variância em modelos mistos desbalanceados, subsídios para uma opção tão confortável quanto possível do método mais adequado para a obtenção das estimativas, em suas pesquisas, o presente artigo apresenta, com ilustrações numéricas, os principais métodos de estimação. Ademais, apresenta, aos usuários não iniciados, conceitos introdutórios necessários para a utilização do PROC MIXED do Sistema Estatístico SAS, na obtenção das estimativas dos componentes de variância através dos métodos ali disponíveis, considerando a ocorrência de diferentes matrizes de variâncias e covariâncias.
\end{abstract}

Palavras-chave: PROC MIXED, SAS, componentes de variância, modelo misto

\section{ESTIMATION METHODS OF VARIANCE COMPONENTS IN UNBALANCED MIXED MODELS}

\begin{abstract}
Scientists have been giving especial attention to the estimation of variance components in mixed models in the last decades. Consequently several estimation methods have been proposed, what makes the choice of one among them difficult. Aiming to subsidize users of estimation methods of variance components in unbalanced mixed models, aiming to make a better choice in obtaining these estimatives this paper presents, using numerical examples, the main methods of estimation. It also helps beginners with the introductory concepts they need to use the PROC MIXED programfrom the SAS Statistical system, to obtain the estimatives of variance components trough the available methods there presented, considering the occurence of different matrices of variance and covariance.

Key words: PROC MIXED, SAS, variance components, mixed models
\end{abstract}

\section{INTRODUÇÃO}

A análise de experimentos com dados desbalanceados tem merecido, nas últimas décadas, atenção especial por parte dos pesquisadores. Ressalta-se, no entanto, que uma maioria significativa de tais estudos tem considerado apenas os modelos para fatores de efeitos fixos. Por outro lado, em muitas situações práticas um ou mais fatores podem estar associados a processos de amostragem, caracterizando os fatores de efeitos aleatórios e, nesse contexto, assumem grande importância os modelos de efeitos aleatórios bem como os modelos de efeitos mistos. De modo geral, três aspectos são fundamentais na discussão dos modelos mistos: a estimação e os testes de hipóteses sobre os efeitos fixos, a predição dos efeitos aleatórios e a estimação dos componentes de variância. Em geral tanto a predição dos efeitos aleatórios quanto a estimação dos efeitos fixos dependem da estimação dos componentes de variância. Além disso, se a estimação dos componentes de variância é feita sobre dados desbalanceados, a estimativa obtida pode ser função do método utilizado. Em outras palavras, diferentes métodos de estimação podem levar, sob desbalanceamento, a diferentes estimativas de um mesmo parâmetro. Dada a importância do tema, muitos métodos têm sido propostos para a estimação dos componentes de variância. Basicamente, estão universalmente consagrados, nove métodos derivados de três conceitos clássicos da estimação estatística: os momentos, a função de verossimilhança e as funções quadráticas. Dentre os métodos derivados dos momentos estão o método de Fisher (1918), ANOVA, e os métodos I, II e III de Henderson (1953); dentre os derivados da função de verossimilhança estão o método da máxima verossimilhança, ML, de Hartley \& Rao (1967) e da máxima verossimilhança restrita, REML, de Patterson \& Thompson (1971) e, dentre aqueles derivados da estimação sobre funções quadráticas estão os métodos dos estimadores quadráticos de norma mínima, MINQUE (Rao, 1971a), de variância mínima, MIVQUE (Rao, 1971b) e iterativo de norma mínima, I-MINQUE, Searle (1987). 
Valério Filho (1983) apresenta um estudo dos métodos da Análise de Variância e dos Métodos I, II e III de Henderson, elucidando aspectos teóricos, aplicando-os a modelos fixos, aleatórios e mistos, para dados desbalanceados. O autor conclui que o método I é uma extensão do método ANOVA e que o método II é uma modificação do método I, visando à aplicação em modelos mistos que não incluam interação entre efeitos fixos e aleatórios. O método III pode ser aplicado tanto a modelos mistos quanto a modelos aleatórios.

Milliken \& Johnson (1984) apresentam uma obra na qual discutem estimativas pontuais e por intervalo, testes de hipóteses sobre efeitos fixos e aleatórios, tanto para dados balanceados como desbalanceados.

Seraphin (1984) faz uma comparação numérica, através de simulação, dos estimadores de componentes de variância obtidos pelo Método III de Henderson, Máxima Verossimilhança e MIVQUE, em dois modelos genéticoestatísticos de cruzamentos, para dados balanceados e desbalanceados, com dois fatores: hierárquico aleatório e fatorial misto com interação.

Searle (1987) apresenta, em seu texto sobre a teoria de modelos lineares para dados desbalanceados, uma breve discussão sobre os métodos de estimação de componentes de variância, em modelos mistos, para dados balanceados e desbalanceados. $\mathrm{O}$ autor discute as vantagens e desvantagens dos métodos de estimação: ANOVA, métodos I, II e III de Henderson, ML, REML, MINQUE e MIVQUE.

Valério Filho (1991) apresenta um estudo comparativo dos métodos para estimação de componentes de variância, disponíveis no PROC VARCOMP: ANOVA, $\mathrm{ML}, \mathrm{REML}, \mathrm{MIVQUE}(0)$, através de simulação de dados, considerando combinações entre valores de variâncias, associadas aos fatores aleatórios em estudo, número de níveis de efeitos fixos e níveis de desbalanceamento.

Fernandez (1991) enfoca o problema de estimativas negativas para componentes de variâncias, discutindo dois procedimentos : adotar o modelo de populações finitas e utilizar o método da REML.

Searle et al. (1992) apresentam uma obra extensa sobre a estimação dos componentes de variância e análise de modelos mistos, tanto para dados balanceados como desbalanceados. Nessa obra, os autores apresentam, também, detalhes no que se refere à teoria dos métodos, além de apresentar ilustrações para cada método.

Barbin (1993) apresenta um trabalho sobre componentes de variância, enfatizando o método ANOVA para modelos balanceados. Apresenta, também, um método prático, o Método de Hicks, para obtenção dos quadrados médios nos modelos balanceados, sugerindo uma modificação nesse método, visando à coerência entre os resultados obtidos em seu estudo e os resultados obtidos através do método dedutivo. O autor apresenta, também, aplicações na determinação do tamanho de amostras e em estudos de melhoramento genético.
Richardson \& Welsh (1995) apresentam duas definições de máxima verossimilhança e máxima verossimilhança restrita robustas, no sentido de limitar a influência das observações outliers sobre as estimativas dos componentes de variância, fazendo aplicações a dados de experimentos biológicos e químicos. Os autores apresentam, ainda, um estudo através de simulação, para investigar as propriedades assintóticas dos estimadores e também para examinar as vantagens dos métodos robustos, então propostos.

Montebelo (1997), discute as idéias básicas para a interpretação das hipóteses mais comumente testadas pelos pesquisadores das ciências aplicadas, além de discutir a utilização de diversos softwares estatísticos, quando da obtenção das estimativas dos componentes de variância para modelos mistos.

Perri (1998), apresenta um trabalho sobre ajuste de modelos mistos de classificação dupla, utilizando os procedimentos GLM, VARCOMP e MIXED do SAS. A autora discute a performance de cada procedimento, concluindo que o PROC MIXED é o procedimento apropriado para a análise de modelos mistos.

Fernandez (1998) apresenta um estudo de simulação, através de um modelo misto com interação, para comparação dos métodos ANOVA, MINQUE, ML e REML, na eficiência em estimar componentes de variância. Para tanto, utiliza o erro quadrático médio e o valor absoluto do viés, como critérios de comparação.

Um fato a ser ressaltado é que, com raras e felizes excessões, como em Barbin (1993), a bibliografia existente está muito mais direcionada ao profissional com fortes conhecimentos em estatística, que ao profissional das ciências aplicadas que, muitas vezes, por opção própria, é apenas usuário das técnicas e dos sistemas computacionais estatísticos.

O presente artigo pretende apresentar de modo simples e conciso, com vistas no usuário das ciências aplicadas, as idéias mais relevantes e ilustrar numericamente os principais métodos de estimação de componentes de variância em modelos mistos desbalanceados.

\section{MATERIAL E MÉTODOS}

Neste estudo, adota-se, para o modelo linear misto a forma matricial descrita em Searle (1987), Searle et al. (1992), lemma (1995), lemma \& Perri (1996), Perri \& lemma (1999), entre outros:

$$
y=X \theta+Z u+\varepsilon,
$$

onde y é o vetor nx1 de observações; $X$ é a matriz do delineamento dos efeitos fixos, de dimensão nxp; $\theta$ é 0 vetor px1 de efeitos fixos desconhecidos; $Z$ é a matriz do delineamento dos efeitos aleatórios, de dimensão mxp; u é o vetor px1 de efeitos aleatórios desconhecidos; $\varepsilon$ é 0 vetor $n \times 1$ de variáveis aleatórias não observáveis, dito vetor dos erros. 
Assumindo-se que os efeitos aleatórios u e $\varepsilon$ têm distribuição Normal com média zero e matrizes de variâncias e covariâncias $\mathrm{G}$ e $\mathrm{I}_{\varepsilon}^{2}$, respectivamente, então o vetor y terá distribuição normal com média $X \theta$ e matriz de variâncias e covariâncias dada por:

$$
\mathrm{V}=\mathrm{ZGZ}+\mathrm{I} \sigma_{\varepsilon}^{2}
$$

Nos modelos mistos tem-se, em geral, interesse na estimação dos efeitos fixos, na predição dos efeitos aleatórios e na estimação dos componentes de variância.Assim, quando se tem um modelo misto, como o dado em (1), com matriz de variâncias e covariâncias dada em (2), a estimação dos efeitos fixos $\theta$ e a predição dos efeitos aleatórios u podem ser obtidas utilizando-se o Método de Estimação de Mínimos Quadrados Generalizados, GLSE: Generalized Least Square Estimation, que conduz ao sistema de equações normais do modelo misto (SAS, 1997):

$$
\left[\begin{array}{cc}
X^{\prime} R^{-1} X & X^{\prime} R^{-1} y \\
Z^{\prime} R^{-1} X & Z^{\prime} R^{-1} Z+G^{-1}
\end{array}\right]\left[\begin{array}{l}
\theta \\
u
\end{array}\right]=\left[\begin{array}{c}
X^{\prime} R^{-1} y \\
Z R^{-1} y
\end{array}\right]
$$

onde as soluções $\theta^{\circ}$ e ũ são obtidas considerando-se os componentes de variância.

No entanto, em (3) é requerido o conhecimento da matriz $\mathrm{V}$ de variâncias e covariâncias, matriz esta que envolve os componentes de variância que se deseja estimar.

Uma alternativa interessante consiste na obtenção de um conjunto de estimativas dos componentes de variância através de algum dos métodos disponíveis e, na utilização dessas estimativas em lugar dos componentes em V, obtendo-se $\hat{V}$.

\section{Método da análise de variância: ANOVA}

Seja $\sigma^{2}$ o vetor dos componentes de variância a serem estimados e seja s um vetor, da mesma ordem de $\sigma^{2}$, de qualquer forma quadrática linearmente independente das observações. Supondo $s=y^{\prime} A y$, para alguma matriz A tal que $X^{\prime} A X=\varnothing$, tem-se as estimativas dos componentes de variância dadas por:

$$
\hat{\sigma}^{2}=\mathrm{C}^{-} \mathrm{s}
$$

para alguma matriz $\mathrm{C}$, tal que $\mathrm{C}=\left\{\operatorname{tr}\left(\mathrm{Z}_{\mathrm{j}}^{\prime} \mathrm{A}_{\mathrm{i}} \mathrm{Z}_{\mathrm{j}}\right)\right\}$

\section{Método I de Henderson}

O Método I de Henderson consiste na obtenção do valor esperado de cada forma quadrática, correspondente à soma de quadrados para dados balanceados. Iguala-se, então, cada forma quadrática à respectiva esperança matemática, obtendo-se um sistema de equações, cuja resolução fornece as estimativas dos componentes de variância.

\section{Método II de Henderson}

O Método II de Henderson consiste em aplicar o Método I ao modelo ajustado para os efeitos fixos, $\mathrm{y}_{\mathrm{a}}=\mathrm{y}-\mathrm{X} \theta^{\circ}=\mu_{0}^{*} 1+\mathrm{Zu}+\mathrm{K} \varepsilon$, onde $\theta^{\circ}=\left(\mathrm{X}^{\prime} \mathrm{X}\right)^{-} \mathrm{X}^{\prime} \mathrm{y}$ é a solução das equações normais para os efeitos fixos do modelo (1), desconsiderando-se a constante $\mu$, K é uma matriz conhecida e os efeitos aleatórios são os mesmos de (1), portanto Zu não se altera. O Método II de Henderson pode ser utilizado para modelos mistos, desde que tais modelos não possuam interação entre efeitos fixos e aleatórios, ou efeitos aleatórios aninhados dentro de efeitos fixos. (Searle, 1987).

\section{Método III de Henderson}

O Método III de Henderson, também chamado método de ajuste de constantes, utiliza as reduções nas somas de quadrados do modelo completo e de submodelos para estimar os componentes de variância, ao invés das somas de quadrados utilizadas pelos Métodos I e II de Henderson. O Método III de Henderson consiste em obter os estimadores dos componentes de variância, construindo um sistema de equações a partir das diferenças entre as reduções nas somas de quadrado do modelo completo e de sub-modelos de interesse, igualando cada diferença à respectiva esperança matemática.

\section{Método da máxima verossimilhança: ML}

O método da máxima verossimilhança consiste em maximizar a função de verossimilhança das observações, em relação aos efeitos fixos e aos componentes de variância, exigindo a suposição de normalidade dos dados. Assumindo que o vetor $\mathrm{y}$ das observações tem média $X \theta$ e matriz de variâncias e covariâncias, $\mathrm{V}$, conforme descrito em (2). Pode-se dizer que se y $\cap \mathrm{N}(\mathrm{X} \theta, \mathrm{V})$, então, a função de verossimilhança de y é dada por:

$$
\mathrm{L}=\mathrm{L}(\theta, \mathrm{V} \mid \mathrm{y})=\frac{\exp \left\{-\frac{1}{2}(\mathrm{y}-\mathrm{X} \theta)^{\prime} \mathrm{V}^{-1}(\mathrm{y}-\mathrm{X} \theta)\right\}}{(2 \pi)^{1 / 2}|\mathrm{~V}|^{1 / 2}}
$$

onde $|\mathrm{V}|$ é o determinante da matriz V. Derivando-se $\delta=\log$ $L$ em relação a $\theta$, em relação a $\sigma_{\varepsilon}^{2}$ e a cada $\sigma_{i}^{2}$ e igualando-se cada derivada parcial a zero, obtém-se 0 sistema de equações:

$$
\left\{\begin{array}{l}
X^{\prime} \tilde{\mathrm{V}}^{-1} X \tilde{\theta}=X^{\prime} \widetilde{\mathrm{V}}^{-1} \mathrm{y} \\
\operatorname{tr}\left(\tilde{\mathrm{V}}^{-1} Z_{\mathrm{i}} Z_{\mathrm{i}}^{\prime} \tilde{\mathrm{V}}^{-1} Z_{\mathrm{j}} Z_{\mathrm{j}}^{\prime}\right) \tilde{\sigma}_{\mathrm{j}}^{2}=\mathrm{y}^{\prime} \tilde{\mathrm{P}} Z_{\mathrm{i}} Z_{\mathrm{i}}^{\prime} \tilde{\mathrm{P} y}
\end{array}\right.
$$

que resolvido iterativamente para $\tilde{\theta}$ e $\tilde{\sigma}_{i}^{2}$, fornece, respectivamente, os estimadores $M L$ para os efeitos fixos e para os componentes de variância. O PROC MIXED do SAS utiliza uma modificação do processo iterativo de Newton-Rhapson para a obtenção das estimativas dos componentes de variância. As estimativas assim obtidas são sempre não negativas, mas viesadas, devido ao fato 
do método não considerar a perda dos graus de liberdade resultante da estimação dos efeitos fixos do modelo. (Searle et al., 1992).

\section{Método da máxima verossimilhança restrita: REML}

No método da máxima verossimilhança restrita, a função de verossimilhança $L$ dada em (5) é dividida em duas partes independentes, uma referente aos efeitos fixos (L") e outra referente aos efeitos aleatórios (L'). Os componentes de variância referentes aos efeitos aleatórios, $\sigma_{\varepsilon}^{2}$ e $\sigma_{i}^{2}$, são estimados pela maximização de L'e os efeitos fixos $\theta$, através da maximização de L". (Patterson \& Thompson, 1971).Os estimadores REML para os componentes de variâncias $\sigma_{i}^{2}$ são obtidos pela resolução do sistema:

$$
\left\{\operatorname{tr}\left(\tilde{\mathrm{P}} \mathrm{Z}_{\mathrm{i}} \mathrm{Z}_{\mathrm{i}}^{\prime} \tilde{\mathrm{P}} \mathrm{Z}_{\mathrm{j}} \mathrm{Z}_{\mathrm{j}}^{\prime}\right) \tilde{\sigma}_{\mathrm{j}}^{2}\right\}_{\mathrm{j}=0}^{\mathrm{r}}=\mathrm{y}^{\prime} \tilde{\mathrm{P}} \mathrm{Z}_{\mathrm{i}} \mathrm{Z}_{\mathrm{i}}^{\prime} \tilde{\mathrm{P} y}
$$

O método da máxima verossimilhança restrita, também é iterativo e exige normalidade, como o Método ML. No entanto, considera a perda dos graus de liberdade devida aos efeitos fixos, fornecendo estimadores não viesados e de variância mínima para dados balanceados. Para dados desbalanceados, ambos os métodos fornecem estimativas viesadas. Entretanto, segundo Searle (1971), Harville (1977), Searle et al. (1992); e Perri \& lemma (1999), dadas as propriedades dos estimadores REML, eles têm sido preferidos quando os dados são desbalanceados.

\section{Estimador quadrático não-viesado de norma mínima: MINQUE}

O método do estimador quadrático não-viesado de norma mínima (MINQUE) não exige normalidade e busca um estimador não viesado de norma mínima, que seja função quadrática das observações. Ressalta-se, porém, que o critério de norma mínima é imposto à matriz núcleo das formas quadráticas utilizadas na estimação das funções quadráticas das observações. Seu desenvolvimento envolve álgebra extensa e seu conceito utiliza um conjunto de pesos pré-atribuídos (w) para os componentes de variância desconhecidos. Assim, segundo Searle et al.(1992), diferentes pesos podem levar a diferentes estimativas para um mesmo conjunto de dados. O método MINQUE fornece estimativas não viesadas, invariantes e de norma mínima para os componentes de variância, fazendo uso de formas quadráticas. Em síntese, a estimação dos componentes de variância pelo método MINQUE consiste na resolução do sistema:

$$
\left\{\operatorname{tr}\left(P_{w} Z_{i} Z_{i}^{\prime} P_{w} Z_{j} Z_{j}^{\prime}\right)\right\} \hat{\sigma}_{j}^{2}=\left\{y^{\prime} P_{w} Z_{i} Z_{i}^{\prime} P_{w} y\right\}
$$

onde $P_{w}$ é o projetor ortogonal dado por $P_{w}=V_{w}^{-1}-V_{w}^{-1} X\left(X^{\prime} V_{w}^{-1} X\right)^{-} X^{\prime} V_{w}^{-1}$ e $V_{w}$ é como em (2), considerando-se os pesos $w$ em lugar de $\sigma^{2}$. Ressaltase, porém, que as estimativas MINQUE não são obtidas iterativamente. Considera-se o primeiro resultado de (8), obtido a partir de um conjunto de pesos iniciais. Se para a obtenção das estimativas pelo método MINQUE, são utilizados como pesos pré-atribuídos (w), as estimativas para os componentes de variância obtidas pelo método ANOVA, então o método MINQUE é denominado $\operatorname{MINQUE}(A)$.

\section{Estimador quadrático não-viesado de variância mínima: MIVQUE}

Quando a variância mínima é o critério estabelecido no lugar de norma mínima e, os critérios de ser função quadrática das observações e ser não viesado são mantidos, tem-se o estimador quadrático não-viesado de variância mínima: MIVQUE.

O estimador MIVQUE $(A)$ utiliza as equações REML sem iteração, tomando as estimativas ANOVA como valores a priori. Embora a teoria MIVQUE exija que os valores a priori sejam independentes dos dados, justificase o uso das estimativas ANOVA em decorrência da facilidade de obtenção. De modo análogo, define-se o estimador MIVQUE(0), como um estimador MIVQUE, com a suposição à priori de que a matriz de variâncias $e$ covariâncias é a matriz identidade. Conforme visto, o método MINQUE não exige qualquer suposição sobre a forma da distribuição da variável aleatória y. No entanto, se a suposição usual de normalidade está presente, a solução MINQUE tem a propriedade de ser uma função quadrática não-viesada das observações com variância mínima, ou seja, é um estimador MIVQUE. (Searle, 1987).

\section{Estimador quadrático não-viesado de norma mínima iterativo: I-MINQUE}

O estimador MINQUE utiliza um vetor de pesos w como um valor pré-fixado de $\sigma^{2}$ e nenhuma iteração está envolvida. No entanto, tendo sido obtida uma solução $\tilde{\sigma}_{1}^{2}$, por exemplo, existe a idéia de utilizá-la como um novo vetor de pesos w, para o qual um novo conjunto de equações pode ser estabelecido e resolvido, produzindo $\tilde{\sigma}_{1}^{2}$, e assim sucessivamente. Isto leva a utilizar as equações MINQUE iterativamente para produzir o MINQUE Iterativo, obtendo os estimadores I-MINQUE. (Searle et al., 1992).

\section{RESULTADOS E DISCUSSÃO}

A ilustração dos métodos descritos neste artigo está feito em duas partes.

A primeira tem como objetivo ilustrar comparativamente, sem o apelo de competição, os nove métodos descritos, de modo a estar tão próximo quanto possível da realidade do pesquisador. Para tanto, através de um conjunto de dados desbalanceados, adaptados de Searle et al. (1992), constantes da TABELA 1, para o qual adota-se o modelo misto de dois fatores com interação, 
descrito em (9), são obtidas as estimativas tomandose uma matriz de variâncias e covariâncias com estrutura do tipo VC, considerada default na maioria dos sistemas estatísticos disponíveis no mercado.

A segunda parte busca ilustrar uma situação mais próxima da ideal, na qual o pesquisador dispõe de meios para estudar a estrutura de covariâncias de seus dados. São ilustrados os três métodos disponíveis no PROC MIXED do SAS: ML, REML e MIVQUE(0), considerandose o modelo dado em (9) e os dados constantes da TABELA 1. Nesse contexto, são consideradas, conforme pode ser observado na TABELA 3, quatro estruturas para as matrizes de variâncias e covariâncias: VC, TOEP(1), CS e HF, dentre cerca de quarenta disponíveis no PROC MIXED do SAS. Seja, então, o conjunto de dados desbalanceados, adaptados de Searle et al.(1992) descrito na TABELA 1.

O modelo utilizado para descrever os dados da TABELA 1 é o modelo misto desbalanceado com dois fatores ( $A$ fixo e $B$ aleatório) e interação $A B$ aleatória, caracterizado por:

$$
\mathrm{y}_{\mathrm{ijk}}=\mu+\alpha_{\mathrm{i}}+\beta_{\mathrm{j}}+(\alpha \beta)_{\mathrm{ij}}+\varepsilon_{\mathrm{ijk}}
$$

onde, para $\mathrm{i}=1,2 ; \mathrm{j}=1,2,3 ; \mathrm{k}=1, \ldots, \mathrm{n}_{\mathrm{ij}}, \mathrm{y}_{\mathrm{ijk}}$ é a k-ésima observação no i-ésimo nível do fator $\mathrm{A}$ e j-ésimo nível do fator $B ; \mu$ é uma constante inerente a todas as observações; $\alpha_{i}$ é o efeito fixo do nível i do fator $A ; \beta$ é o efeito aleatório do nível j do fator $B, \operatorname{com} \beta_{\mathrm{j}} \cap \mathrm{N}\left(0, \sigma_{\beta}^{2}\right)$ e independentes; $(\alpha \beta)_{\mathrm{ij}}$ é o efeito aleatório da interação entre o i-ésimo nível do fator $\mathrm{A}$ e o j-ésimo nível do fator $\mathrm{B}$, com $\gamma_{\mathrm{ij}} \cap \mathrm{N}\left(0, \sigma_{\alpha \beta}^{2}\right)$ e independentes; $\varepsilon_{\mathrm{ijk}}$ é 0 erro aleatório referente à observação $y_{i j k}$, com $\varepsilon_{\mathrm{ijk}} \cap \mathrm{N}\left(0, \sigma_{\varepsilon}^{2}\right)$ e independentes; $\beta_{\mathrm{j}},(\alpha \beta)_{\mathrm{ij}}$ e $\varepsilon_{\mathrm{ijk}}$ são independentes.

\section{Matriz de variâncias e covariancias do tipo VC}

Para a obtenção das estimativas dos componentes de variância para os dados da TABELA 1 , considerando-se o modelo (9), adotou-se uma matriz de variâncias e covariâncias com estrutura do tipo VC, descrita em (10), para os efeitos aleatórios.

TABELA 1 - Dados de um experimento com classificação dupla cruzada.

\begin{tabular}{lllll}
\hline Fator $A$ & \multicolumn{4}{c}{ Fator $B$} \\
\cline { 2 - 4 } & $\mathrm{j}=1$ & $\mathrm{j}=2$ & $\mathrm{j}=3$ \\
\hline \multirow{3}{*}{$=1$} & $\mathrm{y}_{111}=7,9$ & $\mathrm{y}_{121}=6,0$ & $\mathrm{y}_{131}=2,0$ \\
& $\mathrm{y}_{112}=8,1$ & - & - & \\
& $16,0(2) 8,0$ & $6,0(1) 6,0$ & $2,0(1) 2,0$ & $24,0(4) 6,0$ \\
& $\mathrm{y}_{211}=8,0$ & $\mathrm{y}_{221}=4,8$ & $\mathrm{y}_{231}=12,0$ \\
$\mathrm{i}=2$ & - & $\mathrm{y}_{222}=7,2$ & - \\
& $8,0(1) 8,0$ & $12,0(2) 6,0$ & $12,0(1) 12,0$ & $32,0(4) 6,0$ \\
& $24,0(3) 8,0$ & $18,0(3) 6,0$ & $14,0(2) 7,0$ & $56,0(8) 7,0$ \\
\hline
\end{tabular}

Fonte: Searle et al. (1992).

$$
\mathrm{G}=\left[\begin{array}{cc}
\mathrm{I}_{3} \sigma_{\beta}^{2} & \phi \\
\phi & \mathrm{I}_{6} \sigma_{\alpha \beta}^{2}
\end{array}\right]
$$

Assim tem-se $V$ dada em (2) representada por:

$$
\mathrm{V}=\mathrm{ZGZ}+\mathrm{I}_{9} \sigma_{\varepsilon}^{2}=\mathrm{Z}\left[\begin{array}{cc}
\mathrm{I}_{3} \sigma_{\beta}^{2} & \phi \\
\phi & \mathrm{I}_{6} \sigma_{\alpha \beta}^{2}
\end{array}\right] \mathrm{Z}^{\prime}+\mathrm{I}_{9} \sigma_{\varepsilon}^{2}
$$

onde $\mathrm{Z}$ é a matriz de delineamento referente aos efeitos aleatórios do modelo (9).

\section{Estimativas ANOVA}

As estimativas ANOVA para os componentes de variância são obtidas pela resolução do sistema formado pela igualdade das somas de quadrados às respectivas esperanças:

$$
\left[\begin{array}{rrr}
5,2500 & 2,8333 & 2,0000 \\
-0,2500 & 2,1667 & 2,0000 \\
0,0000 & 0,0000 & 2,0000
\end{array}\right]\left[\begin{array}{c}
\hat{\sigma}_{\beta}^{2} \\
\hat{\sigma}_{\alpha \beta}^{2} \\
\hat{\sigma}_{\varepsilon}^{2}
\end{array}\right]=\left[\begin{array}{r}
6,0 \\
42,0 \\
2,9
\end{array}\right] \Rightarrow\left[\begin{array}{c}
\hat{\sigma}_{\beta}^{2} \\
\hat{\sigma}_{\alpha \beta}^{2} \\
\hat{\sigma}_{\varepsilon}^{2}
\end{array}\right]=\left[\begin{array}{r}
-8,6124 \\
17,0524 \\
1,4500
\end{array}\right]
$$

As estimativas obtidas em (12) são estimativas não viesadas para os componentes de variância. Além disso, observa-se que a estimativa obtida para $\hat{\sigma}_{\beta}^{2}$ é negativa, fato que pode causar embaraço ao usuário do método ANOVA, uma vez que, por definição, $\hat{\sigma}_{\beta}^{2}$, variância do fator aleatório $B$, é positiva.

Segundo Searle et al. (1992), uma vez que a única propriedade do estimador ANOVA para os componentes de variância é ser não-viesado, pode ser conveniente renunciar a tal método em função de outros, como o ML, por exemplo, que fornece estimativas viesadas, mas que para grandes amostras têm as propriedades de normalidade e eficiência assintóticas.

\section{Estimativas do tipo Henderson 1}

Para a utilização do Método I de Henderson, considera-se, aqui, o fator fixo A como fator aleatório. Calculam-se, então, as formas quadráticas para o modelo considerado, igualando-as às respectivas esperanças, formando um sistema cuja resolução fornece as estimativas através do Método I de Henderson para os componentes de variância:

$$
\left[\begin{array}{rrrr}
4,0000 & 0,2500 & 1,5000 & 1,0000 \\
0,3333 & 5,2500 & 2,8333 & 2,0000 \\
-0,3333 & -0,2500 & 2,1667 & 2,0000 \\
0,0000 & 0,0000 & 0,0000 & 2,0000
\end{array}\right]\left[\begin{array}{c}
\hat{\sigma}_{\alpha}^{2} \\
\hat{\sigma}_{\beta}^{2} \\
\hat{\sigma}_{\alpha \beta}^{2} \\
\hat{\sigma}_{\varepsilon}^{2}
\end{array}\right]=\left[\begin{array}{r}
8,0 \\
6,0 \\
42,0 \\
2,9
\end{array}\right] \Rightarrow\left[\begin{array}{c}
\hat{\sigma}_{\alpha}^{2} \\
\hat{\sigma}_{\beta}^{2} \\
\hat{\sigma}_{\alpha \beta}^{2} \\
\hat{\sigma}_{\varepsilon}^{2}
\end{array}\right]=\left[\begin{array}{r}
-4,0445 \\
-8,0545 \\
16,4945 \\
1.4500
\end{array}\right]
$$

Novamente, em (13) observa-se a ocorrência de estimativas negativas para as variâncias dos fatores $A$ e $B$. Observa-se, também, que as estimativas dos componentes de variância obtidas pelo Método I de Henderson são próximas das estimativas obtidas através do método ANOVA. Henderson (1953) e Searle (1987) comentam que o Método 
I de Henderson não deve ser utilizado para modelos mistos, mas pode ser adaptado a um modelo misto, considerando-se os fatores fixos, temporariamente, como aleatórios, mas as estimativas assim obtidas para os componentes de variância são viesadas. $O$ conjunto de dados da TABELA 1 é proveniente de um modelo misto. Sendo assim, as estimativas obtidas em (13) são viesadas.

\section{Estimativas do tipo Henderson 2}

Considerando o vetor resposta ajustado para os efeitos fixos, igualam-se as formas quadráticas às respectivas esperanças, obtendo-se um sistema cuja solução fornece as estimativas dos componentes de variância para o modelo ajustado, $\mathrm{y}_{\mathrm{a}}=\mathrm{y}-\mathrm{X} \theta^{\circ}=\mu_{0}^{*} 1+\mathrm{Zu}+\mathrm{K} \varepsilon$.

$\left[\begin{array}{lll}5,2500 & 2,8333 & 2,0000 \\ 0,0000 & 3,6667 & 3,0000 \\ 0,0000 & 0,0000 & 2,0000\end{array}\right]\left[\begin{array}{c}\hat{\sigma}_{\beta}^{2} \\ \hat{\sigma}_{\alpha \beta}^{2} \\ \hat{\sigma}_{\varepsilon}^{2}\end{array}\right]=\left[\begin{array}{c}12,0417 \\ 36,4583 \\ 2,9000\end{array}\right] \Rightarrow\left[\begin{array}{c}\hat{\sigma}_{\beta}^{2} \\ \hat{\sigma}_{\alpha \beta}^{2} \\ \hat{\sigma}_{\varepsilon}^{2}\end{array}\right]=\left[\begin{array}{c}-2,9848 \\ 8,7558 \\ 1,4500\end{array}\right]$

À exceção de $\hat{\sigma}_{\varepsilon}^{2}$, as demais estimativas de (14) diferem das estimativas obtidas pelos métodos ANOVA e I de Henderson. Diz a literatura que o Método II de Henderson pode ser utilizado para modelos mistos, desde que tais modelos não possuam interação entre fatores fixos e aleatórios. O modelo dado em (9) é um modelo misto com interação entre os fatores $A$ fixo e $B$ aleatório. Portanto, a aplicação do Método II de Henderson a tal modelo é inadequada e sendo assim, as estimativas obtidas em (14) são viesadas.

\section{Estimativas do tipo Henderson 3}

A resolução do sistema formado pelas somas de quadrados referentes ao modelo completo e aos submodelos de interesse igualadas às respectivas esperanças, fornece as estimativas através do método III de Henderson para os componentes de variância:

$\left[\begin{array}{ccc}5,0000 & 2,5758 & 2,0000 \\ 0,0000 & 2,4242 & 2,0000 \\ 0,0000 & 0,0000 & 2,0000\end{array}\right]\left[\begin{array}{c}\hat{\sigma}_{\beta}^{2} \\ \hat{\sigma}_{\alpha \beta}^{2} \\ \hat{\sigma}_{\varepsilon}^{2}\end{array}\right]=\left[\begin{array}{c}11,6364 \\ 36,3636 \\ 2,9000\end{array}\right] \Rightarrow\left[\begin{array}{c}\hat{\sigma}_{\beta}^{2} \\ \hat{\sigma}_{\alpha \beta}^{2} \\ \hat{\sigma}_{\varepsilon}^{2}\end{array}\right]=\left[\begin{array}{c}-5,3640 \\ 13,8040 \\ 1,4500\end{array}\right]$

As estimativas obtidas em (15) são não viesadas para os componentes de variância do modelo (9). Observa-se que as estimativas obtidas pelo Método III de Henderson são próximas das estimativas obtidas pelos métodos ANOVA e I de Henderson. Ressalta-se, porém, que tanto o método ANOVA quanto o método III de Henderson são adequados a modelos mistos, fato que não ocorre com os métodos I e II de Henderson.

Uma vantagem na aplicação do método III de Henderson é que tal método está implementado no PROC VARCOMP do SAS, sob a denominação "type 1", viabilizando sua aplicação a modelos mais parametrizados. Contudo, segundo Searle et al. (1992), a utilização de tal método deve ser cautelosa quando aplicada a modelos envolvendo mais de uma interação.
Estimativas de máxima verossimilhança

Utilizando-se o PROC MIXED do SAS, foram obtidas as estimativas:

$$
\left[\begin{array}{c}
\hat{\sigma}_{\beta}^{2} \\
\hat{\sigma}_{\alpha \beta}^{2} \\
\hat{\sigma}_{\varepsilon}^{2}
\end{array}\right]=\left[\begin{array}{l}
0,0000 \\
5,1078 \\
1,4278
\end{array}\right]
$$

As estimativas ML obtidas em (16) para os componentes de variância são sempre não negativas, mas são viesadas, uma vez que o método não considera a perda dos graus de liberdade, resultante da estimação dos efeitos fixos do modelo.

Segundo Searle et al. (1992), apesar das estimativas obtidas pelo método ML serem viesadas, elas são preferidas por alguns autores em relação às estimativas obtidas pelos métodos ANOVA e III de Henderson, graças às suas excelentes propriedades assintóticas.

\section{Estimativas de máxima verossimilhança restrita}

As estimativas obtidas através do PROC MIXED do SAS são dadas por:

$$
\left[\begin{array}{c}
\hat{\sigma}_{\beta}^{2} \\
\hat{\sigma}_{\alpha \beta}^{2} \\
\hat{\sigma}_{\varepsilon}^{2}
\end{array}\right]=\left[\begin{array}{c}
0,0000 \\
8,2152 \\
1,4278
\end{array}\right]
$$

As estimativas obtidas pelo método REML são não viesadas para dados balanceados. Já para dados desbalanceados as estimativas dos componentes de variância são viesadas, embora o método considere a perda dos graus de liberdade devida ao ajuste dos efeitos aleatórios. Observa-se que as estimativas obtidas em (17) são próximas às estimativas $M L$ e diferentes das obtidas pelos demais métodos. Pode ser observado, ainda, que as estimativas nulas obtidas através dos métodos ML e REML, correspondem às estimativas negativas obtidas através dos demais métodos.

Somente os métodos ML e REML fornecem apenas estimativas não negativas para os componentes de variância. Ressalta-se, porém, que as estimativas foram obtidas através do PROC MIXED do SAS, procedimento este que utiliza, para obtenção das estimativas ML e REML, a maximização da função de verossimilhança e verossimilhança restrita, respectivamente, no espaço paramétrico positivo da função, através de uma modificação do Método de Newton.

Conforme citado anteriormente, com base nas propriedades dos estimadores, o método REML tem sido considerado por alguns autores o preferido para estimar componentes de variância de dados desbalanceados. 


\section{Estimativas quadráticas não-viesadas de norma mínima}

Sejam as estimativas ANOVA consideradas como os pesos w pré-fixados para as equações dadas em (8). A resolução do sistema formado resulta nas estimativas dos componentes de variância, do tipo $\operatorname{MINQUE}(A)$ :

$\left[\begin{array}{ccc}3,1640 & 7,8034 & 3,9022 \\ 1,5869 & 3,9022 & 1,9574 \\ 2,2550 & 3,1640 & 1,5869\end{array}\right]\left[\begin{array}{c}\hat{\sigma}_{\beta}^{2} \\ \hat{\sigma}_{\alpha \beta}^{2} \\ \hat{\sigma}_{\varepsilon}^{2}\end{array}\right]=\left[\begin{array}{c}10,2449 \\ 5,3280 \\ 5,1050\end{array}\right] \Rightarrow\left[\begin{array}{c}\hat{\sigma}_{\beta}^{2} \\ \hat{\sigma}_{\alpha \beta}^{2} \\ \hat{\sigma}_{\varepsilon}^{2}\end{array}\right]=\left[\begin{array}{c}-8,2321 \\ 18,3656 \\ 0,8903\end{array}\right]$

As estimativas dadas em (18), obtidas pelo método MINQUE, são não viesadas, invariantes e de norma mínima. Tais estimativas diferem das obtidas pelos demais métodos, sendo próximas somente das estimativas obtidas pelo método ANOVA.

Ressalta-se, porém, que se forem tomados outros valores para $\mathrm{w}$, diferentes de (12), considerando-se 0 mesmo modelo dado em (9) e o mesmo conjunto de dados constantes da TABELA 1 , outras estimativas para os componentes de variância podem ser obtidas.

Em relação aos métodos $M L$ e $R E M L$ as estimativas MINQUE têm a vantagem de não exigir a normalidade dos dados, fato que muitas vezes não ocorre na prática.

\section{Estimativas quadráticas não-viesadas de variância mínima}

Dado que a solução (18) foi obtida utilizando-se as estimativas ANOVA dos componentes de variância, tem-se que (18) é também solução para o método $\operatorname{MIVQUE}(A)$, supondo-se a normalidade dos dados. Sendo assim, tais estimativas além de invariantes, não viesadas e de norma mínima, são também de variância mínima.

Se for adotada a suposição à priori de que a matriz de variâncias e covariâncias dada em (2) é a matriz identidade, forma-se o sistema do qual são obtidas as estimativas dos componentes de variância, pelo método MIVQUE(0):

$$
\left[\begin{array}{ccc}
5,000 & 12,6250 & 6,5000 \\
15,0000 & 6,5000 & 6,5000 \\
6,0000 & 5,0000 & 5,0000
\end{array}\right]\left[\begin{array}{c}
\hat{\sigma}_{\beta}^{2} \\
\hat{\sigma}_{\alpha \beta}^{2} \\
\hat{\sigma}_{\varepsilon}^{2}
\end{array}\right]=\left[\begin{array}{c}
32,00 \\
64,00 \\
50,90
\end{array}\right] \Rightarrow\left[\begin{array}{c}
\hat{\sigma}_{\beta}^{2} \\
\hat{\sigma}_{\alpha \beta}^{2} \\
\hat{\sigma}_{\varepsilon}^{2}
\end{array}\right]=\left[\begin{array}{c}
-5,2245 \\
14,4745 \\
0,7750
\end{array}\right]
$$

O resultado dado em (19) difere em muito das demais estimativas, obtidas pelos métodos ANOVA, I, II, e III de Henderson, ML e REML, sendo mais próximas das estimativas obtidas pelo método $\operatorname{MINQUE}(\mathrm{A})$.

\section{Estimativas quadráticas não-viesadas iterativas de norma mínima}

O método I-MINQUE também utiliza as equações REML, iterativamente, com a vantagem de não exigir a normalidade dos dados. Para os dados da TABELA 1, as estimativas dos componentes de variância obtidas através do método I-MINQUE são como em (18) se os pesos pré- fixados, $w$, forem as estimativas obtidas pelo método MINQUE(A). Ressalta-se que outras estimativas podem ser obtidas, se forem tomados outros valores para w.

\section{Matrizes mais gerais de variâncias e covariâncias}

Visando a ilustração dos três métodos disponíveis no PROC MIXED do SAS, considera-se o conjunto de dados da TABELA 1. A TABELA 2 apresenta um programa com a estrutura default, VC, para a obtenção das estimativas dos componentes de variância, utilizando-se o PROC MIXED do SAS. Para as demais estruturas de G procede-se de modo análogo.

Para a ilustração do PROC MIXED, são consideradas neste estudo, quatro estruturas para a matriz $G$ de variâncias e covariâncias para os efeitos aleatórios do modelo (1) que estão entre as mais comumentes utilizadas em problemas práticos e que estão disponíveis no PROC MIXED. São elas: i) Componentes de Variância, VC, estrutura default para a matriz $\mathrm{G}$, cujo elemento (i, j) é dado por $\sigma_{k}^{2} 1(i=j)$ onde $\mathrm{i}$ corresponde ao k-ésimo elemento e $1(i=j)$ é igual a 1 quando $i=j$, e zero caso contrário; ii) Diagonal, TOEP(1), dada por $\mathrm{I}^{2}$; iii) Simetria composta, CS, cujo elemento (i, j) é $\sigma_{1}^{2}+\sigma^{2} 1(i=j)$ onde $1(i=j)$ é igual a 1 quando $i=j$, e zero caso contrário; iv) Huynh-Feldt, HF, onde os elementos (i, j) são da forma $\left(\sigma_{\mathrm{i}}^{2}+\sigma_{\mathrm{i}}^{2}\right) / 2-\lambda 1(\mathrm{i} \neq \mathrm{j})$ onde os $\lambda$ 's são autovalores positivos associados à matriz de covariâncias e $1(\mathrm{i} \neq \mathrm{j})$ é igual a 1 quando $i \neq j$, e zero caso contrário.

A TABELA 3 descreve a notação e a forma de tais estruturas de $G$.

Um critério para selecionar a estrutura de $\mathrm{G}$ que "melhor" descreve os dados é o critério AIC, Akaikes's Information Criterion, que pode ser usado para comparar modelos com os mesmos efeitos fixos, mas com diferentes estruturas de G. O modelo com maior AIC é considerado o melhor.

TABELA 2 - Programa para o modelo misto com $G=$ VC.

data exemplo;

do $A=1$ to 2 ; do $B=1$ to 3 ; do rep=1 to 2 ;

input y@@; output;

end; end; end;

cards;

7.98 .16 . 2 . 8 . 4.8 7.2 12.;

proc print;

proc mixed method=ml;

class A B;

model $y=A$;

random $B A^{*} B /$ type $=v c$;

proc mixed method=reml;

class A B;

model $y=A$;

random $B A^{*} B /$ type $=v c$;

proc mixed method=mivque0;

class A B;

model $y=A$;

random $B A^{*} B$ / type $=v c$;

run; 
O método ML não forneceu estimativas para os componentes de variância quando selecionou-se as estruturas CS e HF. Quanto às estruturas VC e TOEP(1), observa-se que as estimativas da variância residual são bem diferentes. Quanto à estrutura da matriz $G$ de variâncias e covariâncias, observa-se que ambas as estruturas VC e TOEP(1) possuem AIC semelhantes, sendo o valor de AIC menor para a estrutura TOEP(1). Rigorosamente, pode-se dizer que a $\mathrm{G}=\mathrm{TOEP}(1)$ descreve "melhor" os dados.

Analisando-se a TABELA 5, observa-se que as estimativas da variância residual são muito próximas para as opções TOEP(1), CS e HF, e menor para a opção $\mathrm{G}=$ VC. Observa-se ainda, que as estruturas $\operatorname{TOEP}(1)$ e CS levaram praticamente, às mesmas estimativas para os componentes de variância. Verifica-se através dos valores

TABELA 3 - Estruturas da matriz de variâncias e covariâncias.

Estruturas de G Notação Exemplo da forma

\begin{tabular}{|c|c|c|}
\hline $\begin{array}{l}\text { Componentes de } \\
\text { Variância (Variance } \\
\text { Components) }\end{array}$ & VC & {$\left[\begin{array}{ccccc}\sigma_{\alpha}^{2} & 0 & 0 & 0 & 0 \\
0 & \sigma_{\alpha}^{2} & 0 & 0 & 0 \\
0 & 0 & \sigma_{\beta}^{2} & 0 & 0 \\
0 & 0 & 0 & \sigma_{\beta}^{2} & 0 \\
0 & 0 & 0 & 0 & \sigma_{\beta}^{2}\end{array}\right]$} \\
\hline $\begin{array}{l}\text { Diagonal (Toeplitz } \\
\text { with one band) }\end{array}$ & TOE & {$\left[\begin{array}{ccccc}\sigma^{2} & 0 & 0 & 0 & 0 \\
0 & \sigma^{2} & 0 & 0 & 0 \\
0 & 0 & \sigma^{2} & 0 & 0 \\
0 & 0 & 0 & \sigma^{2} & 0 \\
0 & 0 & 0 & 0 & \sigma^{2}\end{array}\right]$} \\
\hline $\begin{array}{l}\text { Sime tria Composta } \\
\text { (Compound Symmetry) }\end{array}$ & CS & {$\left[\begin{array}{ccccc}\sigma^{2}+\sigma_{1}^{2} & \sigma_{1}^{2} & \sigma_{1}^{2} & \sigma_{1}^{2} & \sigma_{1}^{2} \\
\sigma_{1}^{2} & \sigma^{2}+\sigma_{1}^{2} & \sigma_{1}^{2} & \sigma_{1}^{2} & \sigma_{1}^{2} \\
\sigma_{1}^{2} & \sigma_{1}^{2} & \sigma^{2}+\sigma_{1}^{2} & \sigma_{1}^{2} & \sigma_{1}^{2} \\
\sigma_{1}^{2} & \sigma_{1}^{2} & \sigma_{1}^{2} & \sigma^{2}+\sigma_{1}^{2} & \sigma_{1}^{2} \\
\sigma_{1}^{2} & \sigma_{1}^{2} & \sigma_{1}^{2} & \sigma_{1}^{2} & \sigma^{2}+\sigma_{1}^{2}\end{array}\right]$} \\
\hline $\begin{array}{l}\text { Huynh-Feldt (Huynh- } \\
\text { Feldt) }\end{array}$ & $\mathrm{HF}$ & {$\left[\begin{array}{ccc}\sigma_{1}^{2} & \frac{\sigma_{1}^{2}+\sigma_{2}^{2}}{2}-\lambda & \frac{\sigma_{1}^{2}+\sigma_{3}^{2}}{2}-\lambda \\
\frac{\sigma_{1}^{2}+\sigma_{2}^{2}}{2}-\lambda & \sigma_{2}^{2} & \frac{\sigma_{2}^{2}+\sigma_{3}^{2}}{2}-\lambda \\
\frac{\sigma_{1}^{2}+\sigma_{3}^{2}}{2}-\lambda & \frac{\sigma_{2}^{2}+\sigma_{3}^{2}}{2}-\lambda & \sigma_{3}^{2}\end{array}\right.$} \\
\hline
\end{tabular}

de AIC que a estrutura TOEP(1) para a matriz G é a que "melhor" descreve os dados. Observa-se, porém, que o valor de AIC para tal estrutura não é muito superior ao valor de AIC para as estruturas VC e CS. A estrutura HF, por sua vez, mostrou-se inadequada para a descrição dos dados, com o valor de AIC foi bem superior ao AIC das demais estruturas.

Analisando-se a TABELA 6, observa-se, novamente, que as estruturas $\operatorname{TOEP}(1)$ e CS levaram às mesmas estimativas dos componentes de variância, com pequena vantagem em termos do valor de AIC para a opção $G=\operatorname{TOEP}(1)$. Quanto às estimativas para a variância residual, somente a estrutura $\mathrm{VC}$ forneceu valor discrepante. No entanto, o valor de AIC para tal estrutura mostrou-se semelhante aos valores de AIC para as estruturas TOEP(1) e CS. A estrutura HF, por sua vez, mostrou-se menos adequada que as demais para a descrição dos dados, pois o valor de AIC foi superior ao valor do AIC para as demais estruturas. Sendo assim, a estrutura TOEP(1) para a matriz $G$ é, dentre as opções aqui apresentadas, a que "melhor" descreve os dados. Segundo Wolfinger (1993), a escolha da estrutura de G mais apropriada para a descrição dos dados da TABELA 1 não deve ser exclusivamente baseada em critérios como o AIC. Deve-se também considerar a natureza dos dados e a experiência do pesquisador.

TABELA 4 - Valores das estimativas obtidas pelo método ML.

\begin{tabular}{llll}
\hline & $\operatorname{TOEP}(1)$ & $\mathrm{CS}$ & $\mathrm{HF}$ \\
\hline$\hat{\sigma}_{\beta}^{2}=0$ & $\hat{\sigma}^{2}=0$ &
\end{tabular}

$\hat{\sigma}_{\alpha \beta}^{2}=5,1078$

$$
\hat{\sigma}_{\varepsilon}^{2}=1,4277 \quad \hat{\sigma}_{\varepsilon}^{2}=6,3625
$$

$A I C=-20,9168 \quad A I C=-20,7532$

(*) o processo para obtenção das estimativas ML não convergiu.

TABELA 5 - Valores das estimativas obtidas pelo método REML.

\begin{tabular}{cccc}
\hline VC & $\operatorname{TOEP}(1)$ & $\mathrm{CS}$ & $\mathrm{HF}$ \\
\hline$\hat{\sigma}_{\beta}^{2}=0$ & $\hat{\sigma}^{2}=6,7073$ & $\hat{\sigma}^{2}=6,7052$ & $\hat{\sigma}_{\mathrm{p}}^{2}=6,2848$ \\
$\hat{\sigma}_{\alpha \beta=8,2152}^{2}$ & & $\mathrm{CS}=0$ & $\mathrm{HF}=6,7062$ \\
$\hat{\sigma}_{\varepsilon}^{2}=1,4378$ & $\hat{\sigma}_{\varepsilon}^{2}=1,6720$ & $\hat{\sigma}_{\varepsilon}^{2}=1,6727$ & $\hat{\sigma}_{\varepsilon}^{2}=1,6723$ \\
AIC $=-18,1521$ & $\mathrm{AIC}=-17,9796$ & $\mathrm{AIC}=-18,9796$ & $\mathrm{AIC}=-26,9796$ \\
\hline
\end{tabular}


TABELA 6 - Valores das estimativas obtidas pelo método MIVQUE(0).

\begin{tabular}{cccc}
\hline VC & TOEP(1) & CS & HF \\
\hline$\hat{\sigma}_{\beta}^{2}=0$ & $\hat{\sigma}^{2}=0,7224$ & $\hat{\sigma}^{2}=0,7224$ & $\hat{\sigma}_{\mathrm{p}}^{2}=27,3566$ \\
$\hat{\sigma}_{\alpha \beta}^{2}=14,4745$ & & $\mathrm{CS}=0$ & $\mathrm{HF}=15,3881$ \\
$\hat{\sigma}_{\varepsilon}^{2}=0,7750$ & $\hat{\sigma}_{\varepsilon}^{2}=7,2794$ & $\hat{\sigma}_{\varepsilon}^{2}=7,2794$ & $\hat{\sigma}_{\varepsilon}^{2}=7,2794$ \\
$\mathrm{AIC}=-18,5896$ & $\mathrm{AIC}=-18,2718$ & $\mathrm{AIC}=-19,2718$ & $\mathrm{AIC}=-28,3154$ \\
\hline
\end{tabular}

\section{CONCLUSÕES}

As estimativas obtidas pelos métodos ANOVA, I, II e III de Henderson, para o conjunto de dados proveniente do experimento com dois fatores cruzados e interação são próximas. Tanto o método ANOVA quanto o método III de Henderson são adequados a modelos mistos, fato que não ocorre com os modelos I e II de Henderson. Os métodos derivados dos Métodos dos Momentos fornece algumas estimativas negativas para os componentes de variância, ocasionando embaraço ao pesquisador em situação, onde, por exemplo, estimam-se variâncias que, por definição, são positivas. Somente os métodos ML e REML, por construção, fornecem estimativas não negativas para os componentes de variância. Tais métodos, no entanto, exigem normalidade dos dados, fato que muitas vezes não ocorre com dados reais. Exceto através das propriedades dos estimadores, não se pode concluir sobre um melhor método, mesmo porque os verdadeiros valores dos componentes de variância, para o conjunto de dados adotado para ilustração, são desconhecidos. Poder-se-ia comparar os métodos, por exemplo, se fosse adotado um conjunto de dados simulados, com determinada distribuição e para o qual os componentes de variâncias fossem, à priori, conhecidos. $A$ matriz $G$ de variâncias e covariâncias com estrutura do tipo TOEP(1) foi a que "melhor" descreveu os dados, dentre as utilizadas, independente do método adotado para obtenção das estimativas dos componentes de variância. A experiência do pesquisador associada à natureza dos dados, deve indicar a estrutura mais apropriada dentre as muitas disponíveis nos sistemas computacionais estatísticos.

\section{REFERÊNCIAS BIBLIOGRÁFICAS}

BARBIN, D. Componentes de variância. Piracicaba: ESALQ,USP, 1993. 108p.

FERNANDEZ, D.W.X. Modelos de populações finitas e máxima verossimilhança finita no problema de estimativas negativas para componentes de variância. Piracicaba, 1991. 118 p. Dissertação (Mestrado) - Escola Superior de Agricultura "Luiz de Queiroz", Universidade de São Paulo.

FERNANDEZ, D.W.X. Análise da estabilidade de cultivares de trevobranco e eficiência de métodos de estimação para componentes de variância. Porto Alegre, 1998. 209p. Tese (Doutorado) Univesidade Federal de Porto Alegre.
FISHER, R.A. The correlation between relatives on the supposition of Medelian inheritance. Transactions of the Royal Society of Edinburgh, v.52, p.399-433, 1918.

HARTLEY, H.O.; RAO, J.N.K. Maximum-likelihood estimation for the mixed analysis of variance model. Biometrika, v.54, p.93-108, 1967.

HARVILLE, D.A. Maximum likelihood approaches to variances component estimation and to relates problems. Journal of the American Statistical Association, v.72, p.320-340, 1977.

HENDERSON, C.R. Estimation of variance and variance components. Biometrics, v.9, p.226-252, 1953.

IEMMA, A.F. Análise de variância de dados desbalanceados. In: CONGRESSO BRASILEIRO DE USUÁRIOS DO SAS, 4., Piracicaba, 1995. Anais. Piracicaba: ESALQ, USP, 1995. $111 \mathrm{p}$.

IEMMA, A.F.; PERRI, S.H.V. Procedimentos do sistema estatísticos SAS para análise de modelos mistos. Multiciência, v.1, p.38-58, 1996.

MILLIKEN, G.A; JOHNSON, D.E. Analysis of messy data: designed experiments. New York: Chapman \& Hall, 1984. 1v.

MONTEBELO, M.I.L. Modelos lineares de efeitos mistos: formulação geral e utilização de alguns sistemas computacionais estatísticos. Piracicaba, 1997. 173p. Tese (Doutorado) - Escola Superior de Agricultura "Luiz de Queiroz", Universidade de São Paulo.

PATTERSON, H.D.; THOMPSON, R. Recovery of inter-block information when block sizes are unequal. Biometrika, v.58, p.545-554, 1971.

PERRI, S.H.V. Ajuste de modelos mistos de classificação dupla: procedimentos do sistema estatístico SAS. Piracicaba, 1998. 126p. Tese (Doutorado) - Escola Superior de Agricultura "Luiz de Queiroz", Universidade de São Paulo.

PERRI, S.H.V.; IEMMA, A.F. Procedimento "MIXED" do SAS para análise de modelos mistos. Scientia Agricola, v.56, p.959-967, 1999.

RAO, C.R. Estimation of variance and covariance components - MINQUE Theory. Journal of Multivariate Analysis, v.1, p.257-275, 1971a.

RAO, C.R. Minimum variance quadratic unbiased estimation of variance components. Journal of Multivariate Analysis, v.1, p.445-456, $1971 b$.

RICHARDSON, A.M.; WELSH, A.H. Robust restrict maximum likelihood in mixed linear models. Biometrics, v.51, p.1429-1439, 1995.

SAS INSTITUTE. SAS/STAT software: changes and enhancements through release 6.12. Cary: Statistical Analysis System Institute, 1997. chapter 18: The MIXED procedure.

SEARLE, S.R. Linear models. New York: John Wiley, 1971. $532 p$. 
SEARLE, S.R. Linear models for unbalanced data. New York: John Wiley \& Sons, 1987. 536p.

SEARLE, S.R.; CASELLA, G.; McCULLOCH, C.E. Variance components. New York: John Wiley \& Sons, 1992. 501p.

SERAPHIN, J.C. Comparação numérica de três estimadores de componentes de variância em alguns modelo genéticoestatísticos de cruzamentos. Piracicaba, 1984. 74p. Dissertação (Mestrado) - Escola Superior de Agricultura "Luiz de Queiroz", Universidade de São Paulo.

VALÉRIO FILHO, W.V. Métodos de Henderson para componentes da variância de dados não balanceados. Piracicaba, 1983. 137p. Dissertação (Mestrado) - Escola Superior de Agricultura "Luiz de Queiroz", Universidade de São Paulo.

VALÉRIO FILHO, W.V. Comparação de métodos para estimação de componentes da variância de dados através de simulação de dados. Piracicaba, 1991. 137p. Tese (Doutorado) - Escola Superior de Agricultura "Luiz de Queiroz", Universidade de São Paulo.

WOLFINGER, R. Covariance structure selection in general mixed models. Communications in Statistics Simulation and Computation, v.22, p.1079-1106, 1993.

$\overline{\text { Recebido em } 11} .02 .00$ 